\title{
Temperature and impurity effects of the polaron in an asymmetric quantum dot
}

\author{
Shu-Ping Shan \\ College of Physics and Electromechanics, Fujian Longyan College, Longyan 364012, P.R. China \\ E-mail: ssping04@126.com
}

Ya-Min Liu

College of Physics and Electronic Information, Inner Mongolia Hulunbei'er College, Hulunbei'er 021008, P.R. China

Jin-Lin Xiao

College of Physics and Electronic Information, Inner Mongolia National University, Tongliao 028043, P.R. China

\author{
Received January 14, 2013, revised April 4, 2013
}

\begin{abstract}
We study the temperature and impurity effects of the ground state energy and the ground state binding energy in an asymmetric quantum dot by using the liner combination operator method. It is found that the ground state energy and the ground state binding energy will increase with increasing the temperature. The ground state energy is a decreasing function of the Coulomb bound potential, whereas the ground state binding energy is an increasing one of it.
\end{abstract}

PACS: 71.38.-k Polarons and electron-phonon interactions;

73.63.Kv Quantum dots.

Keywords: asymmetric quantum dot, impurity, polaron, linear combination operator.

\section{Introduction}

With the rapid development of nanofabrication technology, the investigations of quasi-zero-dimensional quantum dots (QDs) have aroused great interest. Due to the small structures of QDs, some physical properties such as optical and electron transport characteristics are quite different from those of the bulk material. Consequently, there has been a large amount of experimental work [1-4] on QDs. Many investigators have studied the properties of the QDs in many aspects by a variety of theoretical methods [5-8]. Since the electron-phonons interaction is enhanced by the geometric confinement, research on the polaron effect has become a main subject in QD system. For example, a quasi-analytical approach to study energy levels of two-electron QD was studied by Hassanabadi [9]. Bondar [10] calculated the percolation and excitonic luminescence in $\mathrm{SiO}_{2} / \mathrm{ZnO}$ two-phase structure with a density of QDs randomly distributed over a spherical surface by using the effective mass method. Employing Landau-Pekar variational theory, Yao et al. [11]. calculated the ground state energy and effective mass in a two- and three-dimensional QD. Based on the rotational wave approximation and the effective mass approximation, Guo et al. [12] investigated the parameter-dependent optical nutation in $\mathrm{PbSe} / \mathrm{CdSe} / \mathrm{ZnS} \mathrm{QD}$. The energy levels structure of two interacting electrons in a parabolic QD under an external magnetic field of arbitrary strength was studied via the asymptotic iteration method by Al-Dossary [13]. With the effective mass approximation and finite barrier potential, Sadegh and Rezaie [14] studied the effect of magnetic field on the impurity binding energy of the excited states in spherical QD. Yu et al. [15] investigated the geometric phase of QDs in the time-dependent isotropic magnetic field using the invariant theory. Using the Green's function technique, the shot noise in the mesoscopic system composed of a QD coupled to ferromagnetic terminals under the perturbation of ac fields was studied by Zhao $\mathrm{et} \mathrm{al}$. [16]. Under the effective mass approximation and modified by the field theory, Liu et al. [17] theoretically described the nonlinear optical properties of the CdSe/ZnS QD quantum well in the vicinity of a spherical metal nanoparticle. Within the spindensity-functional theory, Zhang et al. [18] investigated the electronic properties of a QD formed by the potentials associated with the surface acoustic wave and constrictions. 
Chen et al. [19] studied the temperature dependence of the binding energy of an impurity bound magnetopolaron in a GaAs parabolic QD by using the second-order RayleighSchrödinger perturbation theory. However, the temperature and impurity effects on the ground state energy and the ground state binding energy of strong-coupling polaron in an asymmetry QD has never been studied yet.

Comparing with the methods mentioned above, the linear combination operator method is simple and concise to study the electron-phonon interaction with arbitrary coupling strength (strong- and weak-coupling) [20,21] in the low-dimensional quantum system. Moreover, the properties of the polaron's vibrational frequency are easier to study by this method [22]. In the present paper, we investigated the temperature and impurity effects on the ground state energy and the ground state binding energy of the polaron in an asymmetry QD using the linear combination operator method.

\section{Theoretical model}

We consider a system in which an electron is moving in a polar crystal and interacting with bulk longitudinal optical (LO) phonons. On the basis of the effective mass approximation, the Hamiltonian of the electron-LO phonon interaction system with a hydrogenic impurity at the center can be written as

$$
\begin{aligned}
H=\frac{\mathbf{p}_{\|}^{2}}{2 m}+ & \frac{p_{z}^{2}}{2 m}+\sum_{\mathbf{q}} \hbar \omega_{L O} a_{\mathbf{q}}^{+} a_{\mathbf{q}}+\frac{1}{2} m \omega_{1}^{2} \rho^{2}+\frac{1}{2} m \omega_{2}^{2} z^{2}+ \\
& +\sum_{\mathbf{q}}\left[V_{q} a_{\mathbf{q}} \exp (\mathrm{iq} \cdot \mathbf{r})+h c\right]-\frac{e^{2}}{\varepsilon_{0} r}
\end{aligned}
$$

where $\mathbf{p}\left(\mathbf{p}_{\|}, p_{z}\right), \mathbf{r}(\boldsymbol{\rho}, z), m, \mathbf{q}\left(q_{\perp}, \mathbf{q}_{\|}\right), \alpha, \quad \omega_{1}$ and $\omega_{2}$ stand for the momentum, the coordinate vector, the mass of the electron, the wave vector, the electron-phonons coupling strength, the parallel confinement strength and the perpendicular confinement strength, respectively. $a_{\mathbf{q}}^{+}$and $a_{\mathbf{q}}$ denote the creation and annihilation operators of the LO phonon with the frequency $\omega_{L O} \cdot-e^{2} /\left(\varepsilon_{0} r\right)$ denotes the Coulomb potential between the electron and the hydrogenlike impurity. $V_{q}$ and $\alpha$ in Eq. (1) are

$$
\begin{aligned}
& V_{q}=i\left(\frac{\hbar \omega_{L O}}{q}\right)\left(\frac{\hbar}{2 m \omega_{L O}}\right)^{1 / 4}\left(\frac{4 \pi \alpha}{V}\right)^{1 / 2}, \\
& \alpha=\left(\frac{e^{2}}{2 \hbar \omega_{L O}}\right)\left(\frac{2 m \omega_{L O}}{\hbar}\right)^{1 / 2}\left(\frac{1}{\varepsilon_{\infty}}-\frac{1}{\varepsilon_{0}}\right),
\end{aligned}
$$

where $V$ refers to the volume of the crystal. Using the Fourier expansion to the Coulomb bound potential as follow:

$$
-\frac{e^{2}}{\varepsilon_{0} r}=-\frac{4 \pi e^{2}}{\varepsilon_{0} V} \sum_{\mathbf{q}} \frac{1}{q^{2}} \exp (-\mathrm{iq} \cdot \mathbf{r})
$$

Then, we carry out the unitary transformation to Eq. (1):

$$
U=\exp \left[\sum_{\mathbf{q}}\left(a_{\mathbf{q}}^{+} f_{q}-a_{\mathbf{q}} f_{q}^{*}\right)\right]
$$

where $f_{q}$ and $f_{q}^{*}$ are the variational functions, we introduce the famous linear combination operator:

$$
\begin{aligned}
& p_{j}=\left[\frac{m \hbar \lambda}{2}\right]^{1 / 2}\left(b_{j}+b_{j}^{+}\right) \\
& r_{j}=i\left[\frac{\hbar}{2 m \lambda}\right]^{1 / 2}\left(b_{j}-b_{j}^{+}\right)
\end{aligned}
$$

where $\lambda$ is the variational parameter. The ground state wave function of the system is chosen as

$$
\left|\psi_{0}\right\rangle=|0\rangle_{a}|0\rangle_{b}
$$

$|0\rangle_{a}$ refers to the unperturbed zero phonon state and $|0\rangle_{b}$ denotes the vacuum state of the $b$ operator. To make the calculation simpler, we choose the usual polaron units ( $\left.\hbar=2 m=\omega_{L O}=1\right)$. The ground state energy is obtained in the following form:

$$
E_{0}=\frac{3}{4} \lambda+\frac{1}{\lambda}\left(\frac{2}{l_{1}^{4}}+\frac{1}{l_{2}^{4}}\right)-\alpha \sqrt{\frac{\lambda}{\pi}}-2 \beta \sqrt{\lambda},
$$

where $l_{1}=\sqrt{\hbar / m \omega_{1}}$ and $l_{2}=\sqrt{\hbar / m \omega_{2}}$ are the parallel and the perpendicular confinement lengths and $\beta=$ $=\left(e^{2} / \varepsilon_{0}\right) \sqrt{m /(\pi \hbar)}$ is the Coulomb bound potential. Performing the variational of $E_{0}$ with respect to $\lambda$, we obtain

$$
\lambda^{2}-\left(\frac{2 \alpha}{3 \sqrt{\pi}}+\frac{4}{3} \beta\right) \lambda^{3 / 2}-\left(\frac{8}{3 l_{1}^{4}}+\frac{4}{3 l_{2}^{4}}\right)=0,
$$

where $\lambda$ is the vibrational frequency of the polaron. If $E_{e}$ and $E_{p}$ denote the energies of the uncoupled electron and phonon, respectively, then the ground state binding energy of the strong-coupling impurity polaron is given by

$$
E_{b}=E_{e}+E_{p}-E_{0}=2 \alpha \sqrt{\frac{\lambda}{\pi}}-\frac{1}{\lambda}\left(\frac{2}{l_{1}^{4}}+\frac{1}{l_{2}^{4}}\right)+2 \beta \sqrt{\lambda} .
$$

The mean number of optical phonons around the ground state is

$$
N=\left\langle\psi_{0}\left|U^{-1} \sum_{\mathbf{q}} a_{\mathbf{q}}^{+} a_{\mathbf{q}} U\right| \psi_{0}\right\rangle=\frac{\alpha}{\sqrt{\pi}} \sqrt{\lambda} .
$$

\section{Temperature effects}

At finite temperature, electron-phonon system is no longer entirely in the ground state. The lattice vibrations excite not only the real phonon but also electron in a parabolic potential. According to the quantum statistics theory, the mean number of phonons is 


$$
\bar{N}=\left[\exp \left(\frac{\hbar \omega_{L O}}{k_{B} T}\right)-1\right]^{-1},
$$

where $k_{B}$ is the Boltzmann constant. The value of $\lambda$ relates not only to the $N$ but also to $\bar{N}$. Through the above analysis, we can obtain the relationship between $E_{0}, E_{b}$, $N$ and $T$.

\section{Numerical results and discussion}

In this section, to show more obviously the influence of the temperature and impurity on the properties of the strongcoupling polaron in an asymmetric QD, we perform the numerical calculation. The results are presented in Fig. 1.

Figure 1(a) shows the relationship between the ground state energy $E_{0}$ of the strong-coupling impurity polaron varying with the temperature $T$ and the Coulomb bound potential $\beta$ for fixed $l_{1}=1.2, l_{2}=1.6$ and $\alpha=6.0$. The lines correspond to the cases of the Coulomb bound potential $\beta=0.2$ and 0.3. Figure 1(b) illustrates the ground state binding energy $E_{b}$ as functions of the temperature $T$ and the Coulomb bound potential $\beta$ for fixed $l_{1}=1.2$, $l_{2}=1.6$ and $\alpha=5.5$. The lines correspond $\beta=1.0$ and
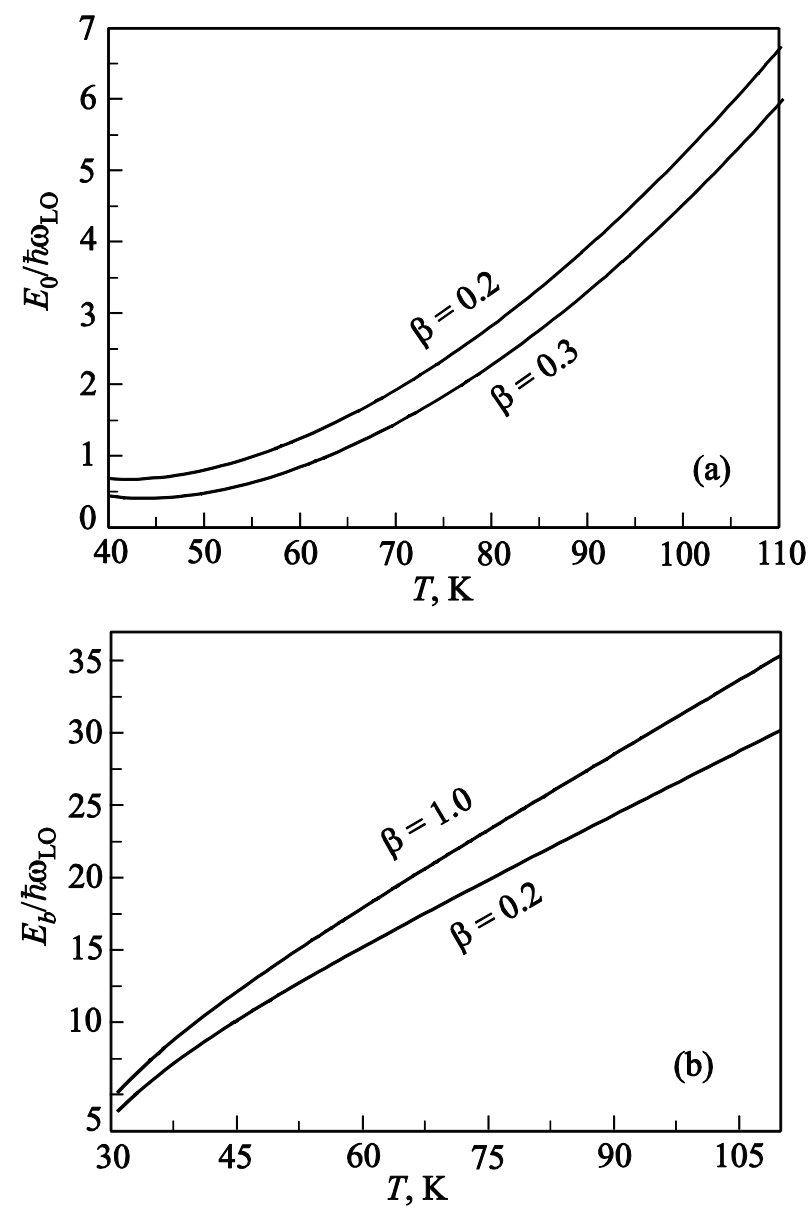

Fig. 1. The relations between the ground state energy $E_{0}$ (a) and ground state binding energy $E_{b}$ (b) and the temperature $T$ at different Coulomb bound potential $\beta$.
0.2. From this figures we can see that the ground state energy and the ground state binding energy increased with increasing temperature. Due to the thermal movement speed of the electron and phonons will be enhanced with rising temperature, so that the electron will interact with more phonons, and the energy of the polaron will be increased. For this reason, the ground state energy and the ground state binding energy increased with the increase of the temperature. From Fig. 1 one can also find that the ground state binding energy is an increasing function of the Coulomb bound potential, whereas the ground state energy is a decreasing one of it. There is Coulomb bound potential between the electron and the hydrogen-like impurity because of the existence of a hydrogen-like impurity in the center of an asymmetric QD. Since the presence of the Coulomb potential is equivalent of introducing another new confinement on the electrons, which leads to greater electron wavefunction overlapping with each other, the electron-phonon interaction will be enhanced. Therefore, the ground state binding energy increases with the rising of the Coulomb bound potential. In Eqs. (8) and (10), we know that the Coulomb bound potential influences the ground state energy with a negative value, but the contribution of the Coulomb bound potential to the ground state binding energy is a positive effect. Hence, we can obtain the results mentioned above. Thus the ground state energy and the ground state binding energy of the asymmetric QDs can be tuned by changing temperature and Coulomb bound potential (impurity).

\section{Conclusion}

In conclusion, based on the linear combination operator method, we have investigated the temperature and impurity effects of the polaron in an asymmetric quantum dot. It is found that the ground state energy and the ground state binding energy will increase with increasing temperature. The ground state energy is a decreasing function of the Coulomb bound potential, whereas the ground state binding energy is an increasing functions of it.

1. J.R. Santos, M.I. Vasilevskiy, and S.A. Filonovich, Phys. Rev. B 78, 245422 (2008).

2. A.I. Yakimov, A.V. Dvurechenskii, G.M. Minkov, A.A. Sherstobitov, A.I. Nikiforov, and A.A. Bloshkin, J. Exp. Theor. Phys. 100, 722 (2005).

3. R.K. Akchurin, I.A. Boginskaya, N.T. Vagapova, A.A. Marmalyuk, and A.A. Panin, Techn. Phys. Lett. 36, 4 (2010).

4. F. Arciprete, M. Fanfoni, F. Patella, A.D. Pia, and A. Balzarotti, Phys. Rev. B 81, 165306 (2010).

5. W. Nomura, T. Yatsui, T. Kawazoe, M. Naruse, and M. Ohtsu, Appl. Phys. B 100, 181 (2010).

6. X.S. Xu, S. Chen, and Y. Toshiki, Sci. China Phys. Mech. Astron. 53, 619 (2010).

7. Z.W. Wang and S.S. Li, J. Appl. Phys. 110, 043512 (2011). 
8. Z.X. Li, Z.H. Ding, and J.L. Xiao, J. Low Temp. Phys. 159, 592 (2010).

9. H. Hassanabadi, Eur. Phys. J. B 74, 415 (2010).

10. N.V. Bondar, Semiconductors 45, 474 (2011).

11. Q.Z. Yao and S.H. Chen, J. Low Temp. Phys. 162, 34 (2011).

12. X.Z. Guo, G.G. Xiong, Q.Q. Xu, M. Xiao, and D.Z. Yao, Appl. Phys. B 103, 189 (2011).

13. O.M. Al-Dossary, Int. J. Theor. Phys. 49, 1187 (2010).

14. E. Sadegh and Gh. Rezaie, Pramana-J. Phys. 75, 749 (2010).

15. Z.X. Yu and Z.Y. Jiao, Int. J. Theor. Phys. 49, 652 (2010).
16. H.K. Zhao, L.L. Zhao, and J. Wang, Eur. Phys. J. B 77, 441 (2010).

17. X.N. Liu and D.Z. Yao, Eur. Phys. J. B 78, 95 (2010).

18. W. Zhang, J. Gao, H.Z. Guo, and C.Y. Zhang, Eur. Phys. J. B 79, 351 (2011).

19. S.H. Chen and J.L. Xiao, Int. J. Mod. Phys. B 21, 5331 (2007).

20. W. Xiao and J.L. Xiao, Int. J. Mod. Phys. B 25, 3485 (2011).

21. C.S. Wang and J.L. Xiao, Mod. Phys. Lett. B 26, 1150003 (2012).

22. W. Xiao and J.L. Xiao, Mod. Phys. Lett. B 23, 2861 (2009). 